\title{
Oligocone trichromacy
}

INSERM

\section{Source}

INSERM. (1999). Orphanet: an online rare disease and orphan drug data base. Oligocone trichromacy. ORPHA:75378

Oligocone trichromacy is a rare non-progressive form of cone photoreceptor dysfunction characterised by reduced visual acuity, normal retinal appearance, absent or reduced cone responses on electroretinog raphy but normal colour vision. 